\title{
EXPERIENCIAS
}

\section{LA TUTORIA COMO RESPUESTA A LAS NECESIDADES DEL ALUMNADO UNIVERSITARIO: UN ESTUDIO EN EL PRIMER CURSO DE ENFERMERÍA DE LA UNIVERSIDAD DE LA LAGUNA}

\author{
THE UNIVERSITY TUTORIAL SYSTEM: A DESCRIPTIVE STUDY OF \\ GUIDANCE NEEDS OF NURSING STUDENTS IN THEIR FIRST YEAR \\ AT THE UNIVERSITY OF LA LAGUNA \\ $M^{a} M$. Árias Hernández, P. R. Álvarez Pérez, $M^{a} C$. García Andrés, \\ J. Cabrera Figueroa, $M^{a}$ D. Martín del Río Aguiar y $M^{a} C$. Robayna Delgado \\ Universidad de La Laguna, Departamento Enfermería, \\ Escuela Universitaria de Enfermería y Fisioterapia
}

\section{RESUMEN}

El alumnado que accede a los estudios universitarios se enfrenta a una realidad compleja en tanto tiene que hacer frente a múltiples situaciones, por lo que se viene insistiendo en los últimos tiempos en la necesidad de implantar programas de tutorización a través de los cuales se ayude a resolver sus dudas y enfrentar sus problemas relacionados con la nueva etapa en la Universidad. Para estructurar dicha ayuda es preciso partir de un análisis de la realidad, de las necesidades y de las demandas que plantean. En este trabajo se presentan los resultados de un diagnóstico inicial de necesidades realizado con el alumnado de primero de Enfermería de la Universidad de La Laguna (Tenerife).

Palabras clave: Orientación universitaria; rol de profesor tutor; diagnóstico de necesidades; estudiantes nuevo ingreso.

\begin{abstract}
University students, mainly in the first years, find themselves having to make decisions about their academic or profesional future from the first moment in which they reach high education. This situation without the adequate aid, makes them feel disoriented and find it difficult to conclude studies in the long term. With this investigation we show a descriptive study of the necessities of orientation that Nursing studients in their first year from the University of La Laguna declare.
\end{abstract}

Key words: University guidance; tutorial role; descriptive study; students in the first year; needs assessment. 


\section{Introducción}

Una de las metas importantes de la institución universitaria actual es ofrecer una enseñanza de calidad que contribuya a reducir el fracaso, abandono y prolongación de los estudios, de modo que se dé respuesta a las demandas socializadoras que plantea la sociedad, preparando personas bien formadas que puedan lograr una incorporación exitosa al mercado de trabajo. Para ello es importante que se conozca en profundidad las demandas, las necesidades, las dificultades, etc. que tienen los estudiantes al inicio y a lo largo de sus estudios, para poder ofertar acciones de mejora que permita el logro de dicha meta.

Entre otras medidas, distintas universidades están poniendo en práctica un sistema de tutorización con el fin de informar a los estudiantes acerca de sus opciones curriculares, hacer un seguimiento de su proceso formativo y desarrollar capacidades para que puedan por sí mismos tomar las decisiones que les resulten más convenientes, evitando de este modo el abandono o fracaso académico.

Como punto de partida previo a dicha intervención tutorial, es necesario llevar a cabo un estudio real de las condiciones que presenta cada contexto, de tal modo que se pueda dar una correlación entre demandas e intervenciones. Este es precisamente el objeto del presente trabajo, en el que se recogen los datos obtenidos en un estudio de necesidades para conocer la opinión de los estudiantes de Enfermería de nuevo ingreso, en la Escuela Universitaria de Enfermería y Fisioterapia de la Universidad de La Laguna, en relación a las dificultades y a la visión que tienen de la Universidad. Se trata, por lo tanto, de un estudio diagnóstico descriptivo, elaborado a partir de las respuestas de una muestra de estudiantes de primer curso, para profundizar en sus percepciones acerca de las necesidades de orientación y las dificultades a las que se enfrentan en su proceso formativo.

\section{La tutoría universitaria o la necesidad de un reajuste de la función docente}

El Informe Universidad 2000 resalta al ocuparse de los sistemas de apoyo al alumnado, la necesidad de crear la figura del profesor tutor del estudiante, entendiéndolo como un servicio de ayuda fundamental de las universidades. Según este planteamiento, una parte del profesorado y una parte del tiempo que se destina a actividades docentes deberá asignarse a tareas de asesoramiento y tutorización a los estudiantes (Informe Bricall, III-54).

La tutoría universitaria tiene una clara función en la atención personalizada al alumnado, facilitando su adaptación e integración en el sistema formativo superior, mejorando el aprovechamiento académico y allanando el camino para que la transición a la vida activa y al trabajo pueda llevarse a cabo de modo efectivo (Álvarez, 2002). Las particularidades de los planes de estudios, así como el incremento de la optatividad y de la libre elección, la diversificación de las vías de acceso y de salida del sistema universitario, ha supuesto para muchos estudiantes un estímulo que amplifica o aumenta sus oportunidades formativas. Ahora bien, para otros, la variedad en la oferta y la decisión en la elección que sustenta buena parte de la actual enseñanza superior puede generar descontrol o desorientación y conducirlos hacia situaciones poco satisfactorias o decepcionantes.

Para intentar dar respuesta a las necesidades del alumnado, se parte de una concepción de la tutoría como acción orientadora a realizar por el profesorado de modo inseparable a su 
función docente, con el fin de ayudarles a resolver las cuestiones que tienen que ver con su desarrollo personal, académico, social y profesional. El rol de tutor universitario quedaría definido por ser un educador en el sentido más amplio del término, preocupándose por fomentar el desarrollo integral del alumnado desde el marco de la propia práctica educativa (Gallego, 1977; Lázaro, 1997; Álvarez y Cabrera, 1997).

\section{Estudio de necesidades de orientación en Enfermería}

En esta investigación se ha llevado a cabo un análisis descriptivo sobre la visión y las necesidades que perciben los estudiantes de nuevo ingreso en el curso 2002-2003 para los estudios de la Diplomatura en Enfermería en la Escuela Universitaria de Enfermería y Fisioterapia de la Universidad de La Laguna. Hemos pretendido conocer la opinión de los estudiantes de nuevo ingreso en relación a las necesidades y a la visión que tienen de la Universidad con el fin de poder articular, a partir de ahí, distintas acciones de carácter tutorial encaminadas a dar respuesta a dichas demandas.

\section{Objetivos del estudio}

Los objetivos de nuestro estudio son los siguientes:

- Analizar los factores que el alumnado ha tenido en cuenta a la hora de elegir titulación.

- Valorar la información y la orientación recibida por el alumnado acerca de la titulación y la Universidad.

- Analizar las dificultades encontradas por el alumnado al llegar a la Universidad.

- Conocer la opinión de los estudiantes acerca de la titulación que están cursando.

- Analizar las expectativas académicas y profesionales del alumnado relacionadas con la titulación que están cursando.

- Conocer las condiciones en las que se lleva a cabo el proceso de estudio por parte del alumnado.

- Conocer la percepción que tiene el alumnado de las tutorías universitarias.

- Determinar las necesidades de orientación personal que tiene el alumnado.

\section{Muestra}

Los 78 estudiantes de primer curso que participaron de manera voluntaria en la investigación fueron mayoritariamente de género femenino (82.1\%), con edades comprendidas entre los 18-20 años (69.2\%) y que reside durante el curso en la vivienda familiar (42\%). De manera mayoritaria, el alumnado participante costea sus estudios a través de la familia (81.1\%); más de la mitad se benefician de becas (57.7\%) y casi su totalidad $(97.48 \%)$ no realiza ningún tipo de actividad remunerada durante los estudios ni se ha planteado la búsqueda de trabajo. 


\section{Instrumentos de recogida de información}

De acuerdo con los objetivos de la investigación se diseñó un cuestionario estructurado en torno a las siguientes dimensiones: percepción de los estudios de Enfermería; estrategias metodológicas y de evaluación; motivación; técnicas de estudio; la tutoría como recurso; necesidades de orientación personal. La prueba constaba de 47 items de respuesta múltiple y una pregunta abierta, con la que se pretendía obtener una valoración más cualitativa de cuantas observaciones acerca de la situación personal y académica quisieran plantear los estudiantes.

El procesamiento de los datos obtenidos se ha llevado a cabo mediante el Programa Estadístico SPSS en su versión 11.5.1 para Windows en español así como análisis de contenido para la pregunta abierta.

\section{Resultados}

\section{Datos académicos}

El alumnado encuestado considera como aspectos que influyeron bastante-mucho en la elección de la carrera los siguientes: la preferencia o gusto por la carrera $(98.7 \%)$; las salidas laborales de la carrera (85.9\%); estar en posesión de habilidades y aptitudes para esta titulación $(71.3 \%)$; la calidad de la formación científica y profesional ofrecida en la carrera $(56.4 \%)$ y la duración de la carrera (44.9\%). La falta de recursos económicos para estudiar otra carrera fuera de la ULL, la facilidad de estos estudios y el hecho de tener un título son señalados como aspectos que han influido nada-poco en el alumnado estudiado para la elección de la carrera.

El 79.5\% del alumnado proviene de centros públicos y acceden a la Universidad, fundamentalmente, desde Bachillerato LOGSE-PAU (55.1\%); el 85\% se encuentra cursando por primera vez estudios universitarios y una mayoría (73\%) estudia la carrera que ha elegido en primera opción.

Al explorar aspectos relacionados con el proceso de información/orientación acerca de la Universidad ofrecida por su centro de formación, el 79.5\% del alumnado manifiesta haber recibido información que ha sido proporcionada fundamentalmente por los profesores $(59 \%)$, tutores $(53.8 \%)$ y orientadores $(53.8 \%)$. Estos agentes informadores producen bastante satisfacción en el 30\% del alumnado.

Como medios de transmisión de la información valorados por el alumnado como satisfactorios para ser utilizados durante la etapa preuniversitaria destacan: las Jornadas de Puertas Abiertas organizadas por la Universidad (64.1\%), las charlas informativas $(57.7 \%)$ y la consulta de libros, folletos, etc. (57.7\%). La publicidad en medios de comunicación o la programación informática no son considerados medios de transmisión de información que produzcan satisfacción entre el alumnado estudiado (45\%). En relación a información específica relacionada con los estudios de Enfermería un alto porcentaje $(75.7 \%)$ manifiesta no obtenerla por parte de su centro de formación, sino que tuvo que buscarla por iniciativa propia $(58.9 \%)$.

Sobre los cauces de participación del alumnado en el ámbito universitario, el 60\% manifiesta tener información acerca de los órganos de representación y deliberación (Claus- 
tro, Junta de Escuela, Consejos de Departamento ...) y el $92 \%$ sobre los servicios que ofrece la Universidad (biblioteca, aulas de informática ...). El 60\% desconoce las actividades culturales y extraacadémicas (deportes, tuna ...) así como las distintas asociaciones de estudiantes $(68 \%)$.

Los estudiantes consideran que las diferencias entre la Universidad y el Instituto son altas (bastante-mucho) en todas las variables a considerar: tiempo de dedicación a los estudios (85.9\%); forma de impartición de clases y de evaluación (74.4\%); relación profesor/alumno (73.1\%); nivel de exigencia (69.3\%) respecto a lo cual un número importante de alumnos opinó que: "hay un nivel de exigencia alto y que esto influye negativamente en el rendimiento académico"; instalaciones y recursos (65.4\%) opinando que: "hay exceso de alumnos por aula, dificultando el correcto seguimiento de la clase" y la relación que se establece con los compañeros (52.6\%) (Tabla 1).

TABLA 1: ¿En qué medida notaste diferencias entre la universidad y el instituto?

\begin{tabular}{|l|c|c|c|c|c|c|c|c|c|c|}
\hline \multirow{2}{*}{ Aspectos a valorar } & \multicolumn{2}{|c|}{ nada } & \multicolumn{2}{c|}{ poco } & \multicolumn{2}{c|}{ bastante } & \multicolumn{2}{c|}{ mucho } & \multicolumn{2}{c|}{ n/c } \\
\cline { 2 - 14 } & Frecuen. & $\%$ & Frecuen. & $\%$ & Frecuen. & $\%$ & Frecuen. & $\%$ & Frecuen. & $\%$ \\
\hline Nivel de exigencia & 1 & 1.3 & 23 & 29.5 & 29 & 37.2 & 25 & 32.1 & 0 & 0.0 \\
\hline Forma impartir clases & 1 & 1.3 & 19 & 24.4 & 35 & 44.9 & 23 & 29.5 & 0 & 0.0 \\
\hline Formas de evaluar & 2 & 2.6 & 17 & 21.8 & 34 & 43.6 & 24 & 30.8 & 1 & 1.3 \\
\hline Relación profesor-alumno & 1 & 1.3 & 19 & 24.4 & 27 & 34.6 & 30 & 38.5 & 1 & 1.3 \\
\hline $\begin{array}{l}\text { Relación con los } \\
\text { compañeros }\end{array}$ & 10 & 12.8 & 27 & 34.6 & 24 & 30.8 & 17 & 21.8 & 0 & 0.0 \\
\hline Instalaciones y recursos & 4 & 5.1 & 23 & 29.5 & 36 & 46.2 & 15 & 19.2 & 0 & 0.0 \\
\hline $\begin{array}{l}\text { Tiempo de dedicación a } \\
\text { los estudios }\end{array}$ & 2 & 2.6 & 8 & 10.3 & 19 & 24.4 & 48 & 61.5 & 1 & 1.3 \\
\hline
\end{tabular}

\section{Percepción de los estudios de Enfermería (o sobre la titulación)}

Al estudiar el grado de dificultad que el alumnado de nuevo ingreso ha encontrado durante los primeros meses del curso podemos constatar que más del $60 \%$ de los encuestados consideran aspectos de gran dificultad y, por orden de importancia, los siguientes: la distribución horaria respecto a lo cual la mayoría de alumnos que aportan sugerencias consideran: "el número de horas lectivas es excesivo", "los horarios de clase son demoledores", la adaptación al ritmo de la universidad, el horario de secretaría, el servicio de comedor y cafeterías sobre el que mayoritariamente desean que se lleve a cabo una reestructuración importante y el servicio de secretaría. No han encontrado dificultades en aspectos relacionados con el servicio de bibliotecas, alojamiento o vivienda, presencia de barreras arquitectónicas $\mathrm{y}$ en el servicio de transporte.

Un elevado porcentaje del alumnado manifiesta que desconoce los procesos administrativos relacionados con cambios de titulación (85.9\%); traslado de expedientes $(74.4 \%)$ y convalidaciones; sin embargo, afirman tener conocimientos acerca de aspectos relacionados 
con el número de convocatorias anuales y el número de asignaturas que debe aprobar para no perder la matrícula (Tabla 2).

TABLA 2: ¿Conoces los procesos administrativos y los requisitos propios de la titulación?

\begin{tabular}{|l|c|c|c|c|c|c|}
\hline \multirow{2}{*}{\multicolumn{1}{|c|}{ Aspectos a valorar }} & \multicolumn{2}{c|}{ sí } & \multicolumn{2}{c|}{ no } & \multicolumn{2}{c|}{ n/c } \\
\cline { 2 - 8 } & Frecuen. & \% & Frecuen. & \% & Frecuen. & \% \\
\hline Convalidaciones & 19 & 24.4 & 57 & 73.1 & 2 & 2.6 \\
\hline Traslados de expediente & 18 & 23.1 & 58 & 74.4 & 2 & 2.6 \\
\hline Cambios titulación & 9 & 11.5 & 67 & 85.9 & 2 & 2.6 \\
\hline $\begin{array}{l}\text { Número asignaturas que debes } \\
\text { aprobar para no perder matrícula }\end{array}$ & 49 & 62.8 & 29 & 37.2 & 0 & 0.0 \\
\hline Número convocatorias anuales & 55 & 70.5 & 21 & 26.9 & 2 & 2.6 \\
\hline
\end{tabular}

Más de la mitad de los encuestados valoran como aspectos positivos de la carrera las notas obtenidas. Estas producen bastante-mucha satisfacción al $85.9 \%$ de los encuestados; también la relación con los compañeros (78.2\%); los contenidos teóricos que reciben (64.1\%); el plan de estudios (57.7\%); la relación profesor-alumno y la competencia del profesor. Consideran aspectos negativos de la carrera y, por orden de importancia, las horas de permanencia en el centro (94.8\%) ante lo cual manifiestan de manera explícita: "que se mejoren los horarios", las características físicas del aula (85.9\%) de las que se opina que: "la infraestructura no es adecuada", la organización de la escuela, las formas de evaluar, la metodología o forma de dar las clases, las instalaciones y recursos (baños, vestuarios ...) y, en último lugar, el nivel de exigencia establecido en los estudios (52.6\%).

\section{Estrategias metodológicas y de evaluación}

Al investigar sobre la metodología docente utilizada en el primer curso de Enfermería obtenemos que la clase teórica de tipo magistral es señalada por la gran mayoría del alumnado $(75.6 \%)$ como el método de enseñanza que se pone en práctica con mayor frecuencia. Esta metodología es valorada por más de la mitad de los encuestados (59.89\%) como poco satisfactoria. Algunos estudiantes opinan al respecto: "la metodología didáctica no es adecuada", "que las clases deberían ser más dinámicas y amenas", y reclaman: "más prácticas en todas las asignaturas". Los debates, las clases teórico-participativas y las tutorías en despacho son consideradas por un elevado porcentaje del alumnado encuestado (alrededor del $60 \%$ ) como métodos de enseñanza bastantes satisfactorios que se ponen en práctica esporádicamente.

En relación a las metodologías de evaluación utilizadas se señala que los exámenes con ejercicios y problemas constituye el método de evaluación predominante en el primer curso de Enfermería; más de la mitad (56.4\%) valora esta metodología como poco satisfactoria y dice de ella que: "debería cambiarse el tipo de examen por considerar que no es el sistema de evaluación más adecuado”. Otras metodologías de evaluación son, por orden de impor- 
tancia: los trabajos grupales, los exámenes escritos de preguntas abiertas, los exámenes orales, los exámenes de pruebas objetivas o test y la asistencia a clase. Estos métodos son valorados satisfactoriamente por más de la mitad del alumnado (Gráfico 1).

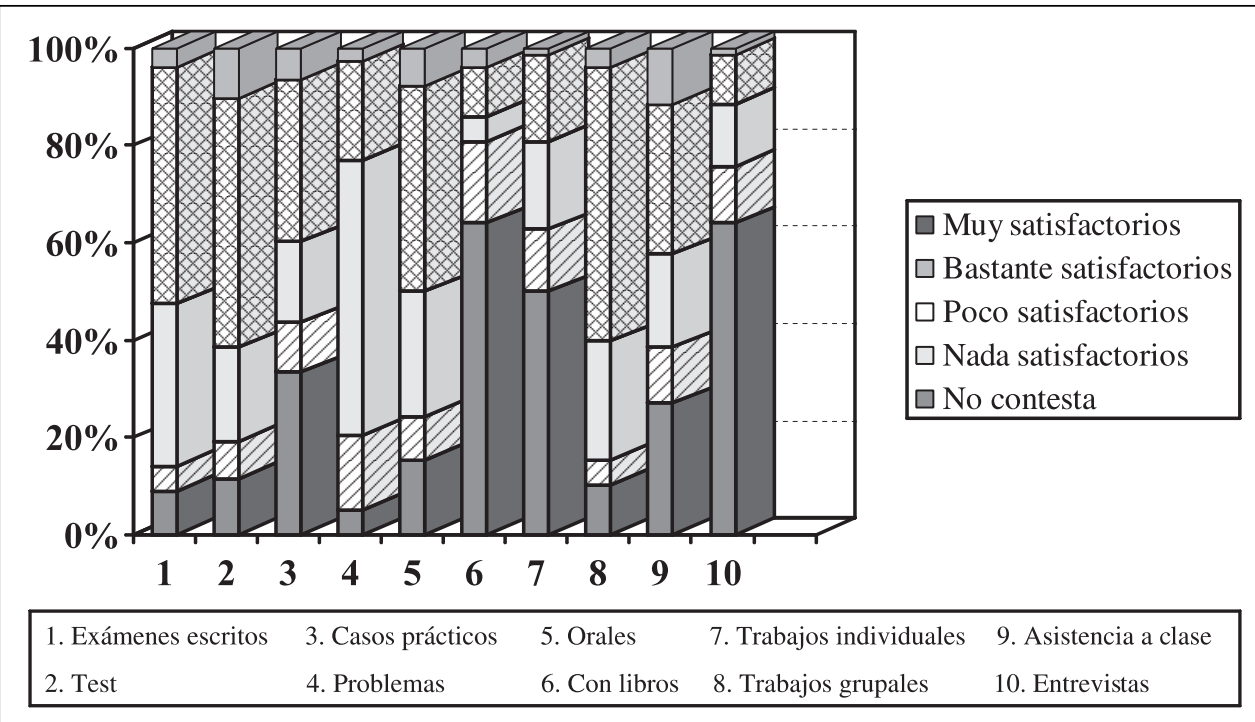

\section{GRÁFICO 1. \\ Metodologías de evaluación.}

En relación a los aspectos en los que se hace hincapié durante la evaluación, un elevado porcentaje (78.4\%) señala que los aspectos memorísticos son los más evaluados, seguido de la valoración del trabajo personal $(60.3 \%)$ — como el plan de cuidados, los ejercicios de resolución de casos etc.- - Aproximadamente la mitad de los encuestados consideran que durante la evaluación no se hace hincapié en los siguientes aspectos: capacidad de síntesis, aplicación de la teoría a situaciones distintas, resolución de problemas y capacidad de expresión y comunicación (Gráfico 2).

\section{La motivación}

En relación al nivel de motivación que despierta los estudios de Enfermería en el alumnado, el $89.8 \%$ manifiesta estar bastante-muy motivado y un $10.3 \%$ refiere presentar pocanada motivación por los estudios que comienza a cursar.

El $69.2 \%$ del alumnado encuestado no se ha planteado abandonar sus estudios; un $30.8 \%$ sí lo ha hecho. El alumnado que se ha planteado abandonar sus estudios considera como factores importantes, motivadores de esta decisión, el nivel de exigencia de los estudios $(19.2 \%)$; porque no cubre sus expectativas $(17.9 \%) \mathrm{y}$, las calificaciones obtenidas $(16.7 \%)$. En menor medida, consideran factores determinantes del abandono de sus estudios 


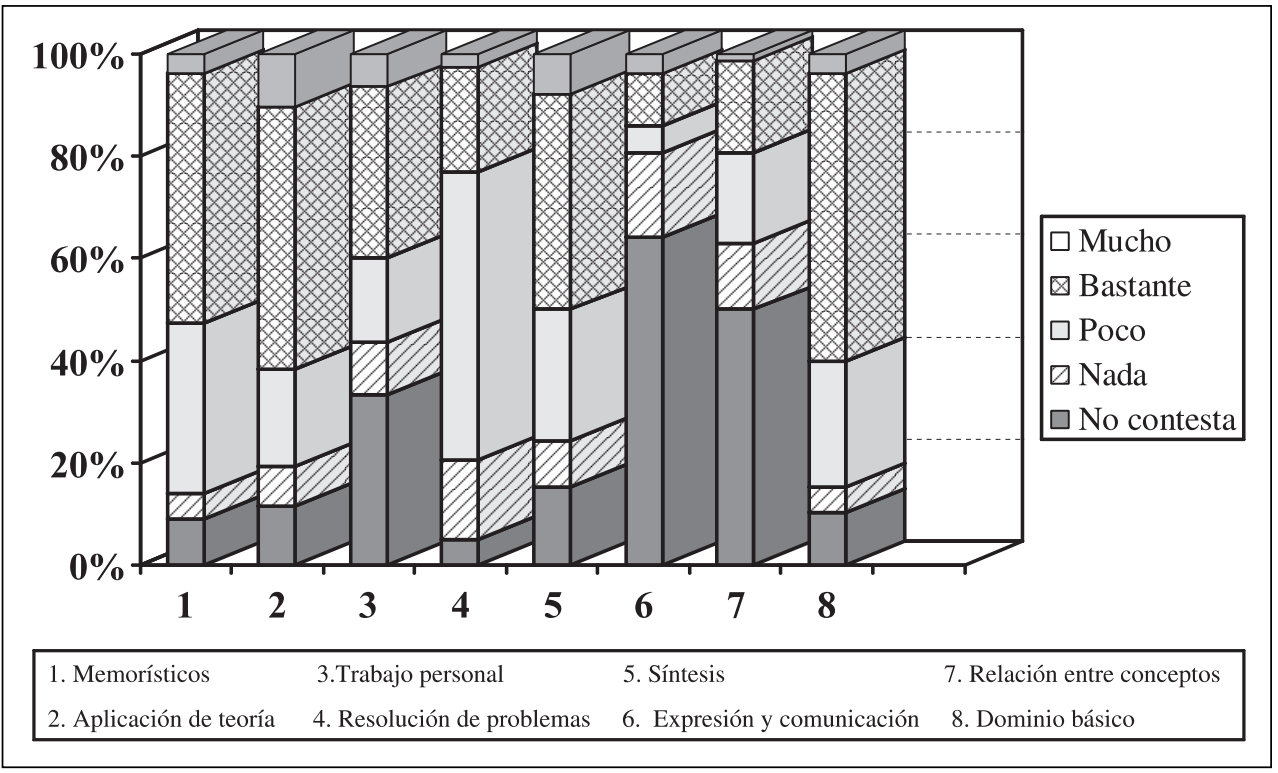

GRÁFICO 2.

Aspectos de la evaluación.

los motivos personales, por cambiar de carrera, los motivos laborales, y los motivos relacionados con sus compañeros (Gráfico 3).

Al preguntar por el tiempo que considerarían necesario para finalizar sus estudios el $52 \%$ creen que lo finalizarán dentro del plazo de duración de los mismos, frente al $42.3 \%$ que considera que necesitará un año más de lo que dura la carrera, motivo por el cual algunos estudiantes manifestaron el deseo de que se llevara a cabo un cambio en el plan de estudios sugiriendo: "poner un año más", "todo está muy condensado" u opiniones del tipo: "recibimos clases que no son útiles".

\section{Técnicas de estudio}

Al estudiar las capacidades y actitudes frente al estudio hemos podido constatar que un elevado porcentaje de alumnado utiliza con mucha frecuencia los apuntes fotocopiados $(85.9 \%)$ y los apuntes dictados por el profesor en clase $(80.7 \%)$. Casi el 30\% dedica más de 20 horas semanales al estudio - excluyendo la asistencia a clase- y emplean habitualmente la habitación personal y la biblioteca como lugar de estudio.

Aproximadamente la mitad del alumnado (48.7\%) manifiesta preocuparse por completar y/o ampliar la formación que recibe en clase. Realizan, principalmente, ampliaciones bibliográficas (37.2\%) y lecturas afines (30.8\%) al temario y, en menor medida, asisten a cursos, seminarios y conferencias (10.3\%). Es de destacar que, ningún alumno-a encuestado-a señaló la estancia en otra universidad, la colaboración con asociaciones profesionales o de 


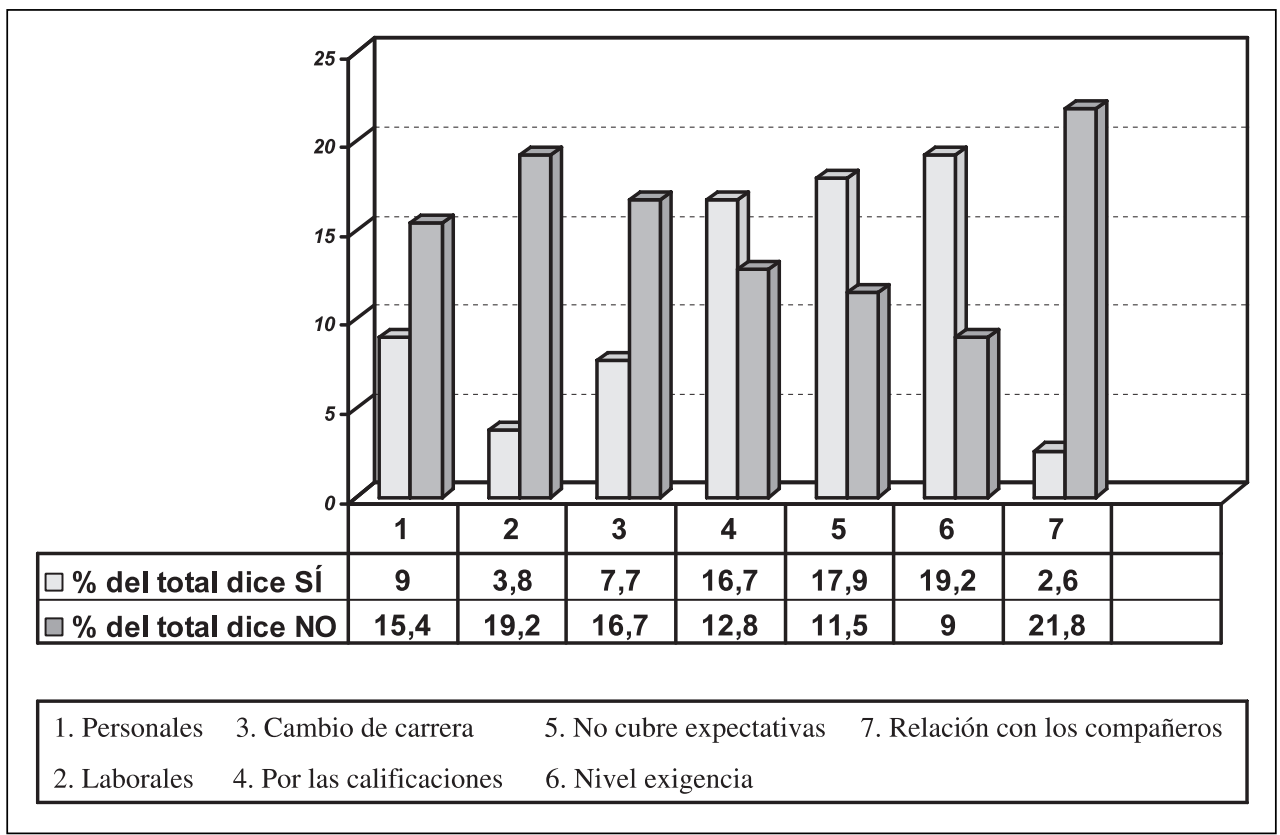

GRÁFICO 3.

Razones para plantearse el abandono.

estudiantes o el realizar trabajos por su cuenta relacionados con la profesión como una opción a utilizar para completar y/o ampliar su formación.

El análisis de los datos referidos al hábito de preparación de los exámenes revela que el $59 \%$ afirma que lo hace en un período de tiempo comprendido entre 10 y 15 días antes de la fecha; sólo un 17.9\% estudia todos los días (Gráfico 4).

En relación a las capacidades y actitudes frente al estudio, la mayoría (alrededor del $60 \%$ ) considera que posee, por orden de importancia, la capacidad de síntesis, de iniciativa/ dinamismo; de planificación y organización del estudio, de comunicación y de constancia en el trabajo. Es de señalar la dicotomía que detectamos entre el elevado nivel de autoestima del alumnado, que considera que tienen bastante-mucha capacidad frente a la planificación y organización del estudio (65.4\%) y la constancia en el trabajo (62.9\%) y el bajo índice de sujetos que manifiesta que organiza el material de estudio para que resulte interesante y comprensible (44.9\%) y aquel que refiere que estudia diariamente, sólo el $17.9 \%$.

\section{La tutoría como recurso}

Un análisis global de los datos obtenidos parece indicarnos que la tutoría no es un recurso demasiado utilizado por el alumnado de Enfermería durante el primer curso. Aunque el 53.8\% del alumnado entrevistado afirma haber hecho uso de la tutoría algu- 


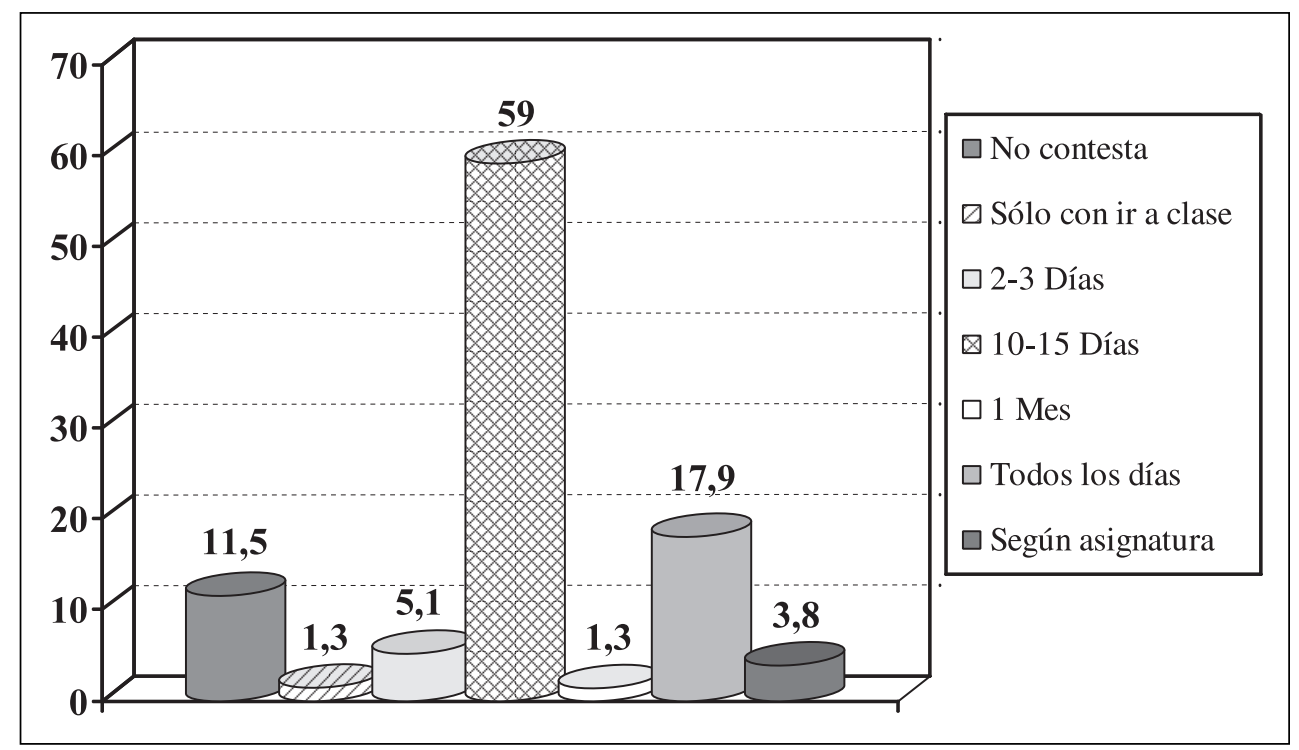

GRÁFICO 4.

Preparación exámenes.

na vez, lo que tratándose de estudiantes de primer curso, con poca experiencia en la Universidad y cierto desconocimiento del modelo de tutoría universitario, puede considerarse una cifra aceptable, lo cierto es que otros datos explorados nos indican un uso muy limitado tanto en la cantidad de veces que acuden a la tutoría, como en las finalidades de su uso.

En general, el alumnado prácticamente no utiliza la tutoría durante el primer período de esta titulación. De los treinta y seis estudiantes que refieren haber usado la tutoría en algún momento durante el primer año académico, la mayoría no asiste a principio de curso, ocho estudiantes refieren hacer "poco" uso de este recurso en este espacio de tiempo y sólo seis consideran que la han usado "bastante" o "muchas" veces a principio de curso. Respecto a su uso a lo largo de todo el curso, observamos como la frecuencia se incrementa ligeramente aunque los datos indican que su uso sigue siendo muy limitado (Tabla 3 ).

Cuando preguntamos al alumnado sobre el uso de la tutoría en relación con la evaluación, encontramos que un $37.1 \%$ afirma acudir a la tutoría antes de los exámenes, de éstos un $12.8 \%$ afirma hacerlo pero pocas veces, el 19.2 afirma haberla usado bastante y sólo un $5.1 \%$ considera que las usa mucho. El uso de la tutoría después de la evaluación sigue una tónica similar, un $32 \%$ acude o usa la tutoría y de éstos, el $20.5 \%$ considera que lo hace "poco" y el 14.1\% afirma hacerlo "bastante".

Otro aspecto estudiado en relación con el uso que los estudiantes han hecho de la tutoría tiene que ver con la finalidad por la que han decidido acudir. Los datos obtenidos parecen dejar claro que lo que ha buscado el alumnado de primer curso de enfermería cuando utilizó la tutoría responde sobre todo a necesidades académicas, concretamente al seguimiento de 
TABLA 3: ¿En qué medida utilizar las tutorías?

\begin{tabular}{|l|c|c|c|c|c|c|c|c|c|c|}
\hline \multirow{2}{*}{ Aspectos a valorar } & \multicolumn{2}{|c|}{ nada } & \multicolumn{2}{c|}{ poco } & \multicolumn{2}{c|}{ bastante } & \multicolumn{2}{c|}{ mucho } & \multicolumn{2}{c|}{ n/c } \\
\cline { 2 - 14 } & Frecuen. & $\%$ & Frecuen. & $\%$ & Frecuen. & $\%$ & Frecuen. & $\%$ & Frecuen. & $\%$ \\
\hline Al principio del curso & 22 & 28.2 & 8 & 10.3 & 4 & 5.1 & 2 & 2.6 & 42 & 53.8 \\
\hline A lo largo del curso & 7 & 9.0 & 22 & 28.2 & 6 & 7.7 & 1 & 1.3 & 42 & 53.8 \\
\hline $\begin{array}{l}\text { Inmediatamente antes } \\
\text { de los exámenes }\end{array}$ & 7 & 9.0 & 10 & 12.8 & 15 & 19.2 & 4 & 5.1 & 42 & 53.8 \\
\hline $\begin{array}{l}\text { Después de la } \\
\text { evaluación }\end{array}$ & 9 & 11.5 & 16 & 20.5 & 11 & 14.1 & 0 & 0.0 & 42 & 53.8 \\
\hline
\end{tabular}

asignaturas (aclarar dudas o revisar exámenes). De los estudiantes de primer curso de enfermería que acuden a tutoría: el $39.7 \%$ ha acudido para revisar exámenes, el 46.2 lo ha hecho para consultar dudas sobre contenidos de las asignaturas, el 7.7 lo ha hecho con la finalidad de aclarar dudas sobre aspectos administrativos y sólo un $2.6 \%$ lo ha hecho para consultar problemas personales.

También hemos querido conocer las preferencias de los estudiantes respecto a la persona que desempeñe el papel de tutor-a. Las opciones sobre las que han podido opinar son tres: la posibilidad de que sea un compañero/a de cursos superiores, un profesor/a de los que le imparte clase o un profesor/a que no le imparte clase. Los resultados demuestran que sólo el $14.1 \%$ elegiría la primera opción, un $80.8 \%$ elegiría al profesor que imparte la materia y el $5.1 \%$ a un profesor/a que no le imparta la materia. Estos resultados de alguna manera son coherentes con la utilidad que, a partir de las respuestas de ítem anteriores, deducimos que asignan a la tutoría, es decir, un recurso que usan puntualmente para el seguimiento de algunas asignaturas.

en futuras investigaciones o proyectos.

\section{Conclusiones}

Después de analizar los distintos apartados de esta investigación y valorar cada una de las variables objeto de estudio, podemos extraer las siguientes conclusiones y consideraciones de carácter general:

1. Destacar que un alto porcentaje del alumnado de nuevo ingreso en los estudios de Enfermería durante el curso 2002-2003 eligió esta carrera en primera opción, lo que constituye un elemento importante que tiene una repercusión en la actitud y motivación hacia los estudios. Aquellos estudiantes que se ven casi forzados a matricularse en carreras que no figuraban entre sus prioridades manifiestan luego, en muchos casos, una actitud desfavorable que incide de manera negativa en la implicación, participación y rendimiento académico, llegando en muchos casos a ser un determinante de los procesos de abandono y/o prolongación de los estudios. En nuestro caso, el hecho de que el $73 \%$ del alumnado haya elegido estos estudios en primera opción es una condición favorable y un factor importante para el éxito académico y para que 
concluyan sus estudios en el tiempo estipulado. No obstante, esto contrasta con el hecho de que un $42 \%$ señale que necesitará tiempo adicional para concluir sus estudios. El desconocimiento de un perfil de carrera claro, de sus itinerarios formativos, incluso de los recursos de apoyo con los que cuenta pueden llevar a los estudiantes a dudar de si serán capaces de responder a las exigencias de la carrera

2. Los estudiantes parecen acusar el cambio de nivel formativo y las diferencias existentes entre la enseñanza secundaria y la universitaria. Desde las horas de permanencia en la Escuela, en el aula, las forma de evaluar, etc. constituyen elementos propios de la enseñanza superior que llevan a acentuar las dificultades de adaptación a este nuevo contexto formativo. Esto no hace sino poner de manifiesto también, no sólo la necesidad de establecer mejores canales de comunicación y cooperación entre los diferentes niveles de la enseñanza y, en este caso concreto, entre la secundaria y la superior, sino también la importancia que tienen los procesos de asesoramiento al alumnado para que su transición e incorporación a la nueva etapa formativa sea satisfactoria. Es preocupante que, en el caso que nos ocupa, el 78\% del alumnado desconociera el plan de estudios en el que se había matriculado y evidencia a las claras una deficiente orientación académica.

3. En general, el alumnado parece valorar de manera poco satisfactoria elementos clave de su proceso formativo. Tanto la metodología didáctica (clases teóricas y expositivas) como la metodología de evaluación (métodos cuantitativos basados en el examen y la resolución de problemas y priorización de los aspectos memorísticos a la hora de evaluar) no parecen ser del agrado de los estudiantes. Procedería en su caso, y dentro de los cauces que se estimen oportunos, iniciar un proceso de reflexión sobre la propia práctica docente, que tendría como finalidad ir introduciendo nuevas perspectivas en la docencia, máxime ahora que en la Universidad parece que se va a imponer un modelo basado en el aprendizaje del alumnado, frente al modelo que de manera mayoritaria ha imperado hasta ahora, basado en la enseñanza del profesor.

4. Destacar que el alumnado manifestó hacer un escaso uso de las tutorías individualizadas y que, cuando hacían uso de éstas, solía ser en los períodos inmediatamente anteriores a los exámenes. Es preciso animar a los estudiantes a usar este recurso, ya que en muchas ocasiones el alumnado saca provecho de algunos de los sistemas de apoyo con que cuenta, no porque no los considere válidos, sino porque desconoce su existencia o los fines para los que fueron creados.

5. Es preciso desmontar algunos de los estereotipos que pesan sobre el uso de la tutoría universitaria (sólo sirve para consultar notas, para recoger trabajos, para hacer la pelota al profesor, etc.). Sería conveniente, en estudios posteriores, explorar el concepto que tanto el alumnado de nuevo ingreso como el profesorado posee acerca de la tutoría universitaria, para poder llegar a entenderla como una actividad de acompañamiento que realiza un profesor con un grupo de estudiantes, complementaria a la docencia, mediante la cual se persigue el seguimiento directo de la actividad académica, personal y profesional de los mismos a lo largo de los estudios universitarios, con el fin de facilitarles una óptima adaptación a la institución, ayudarles a trazar planes de carrera satisfactorios y apoyarles en la toma de decisiones. 


\section{Referencias bibliográficas}

Álvarez, P. (2002). La función tutorial en la universidad; una apuesta por la mejora de la calidad de la enseñanza. Madrid: EOS.

Álvarez, P. y Cabrera, L. (1997). "La acción tutorial en el ámbito universitario; algunas consideraciones planteadas desde un modelo de trabajo colaborativo". VIII Jornadas Nacionales de la Asociación Española de Orientación y Psicopedagogía. Valencia, 268-270.

Bricall, J. M. y cols. (2000). "Informe Universidad 2000". Revista Vivat Academia, 16. Madrid: UAH.

Gallego, S. (1977). "Las funciones del tutor universitario. La Orientación Educativa la Intervención Psicopedagógica integradas en el curriculum". VIII Jornadas Nacionales de la Asociación Española de Orientación y Psicopedagogía. Valencia, 289-292.

Lázaro, A. (1997). "La acción tutorial de la función universitaria". En P. Apocada y C.Lobato (eds). Calidad en la Universidad: Orientación y Evaluación. Barcelona: Laertes.

Fecha de recepción: 15-10-04

Fecha de revisión: 19-09-05

Fecha de aceptación: 20-04-05 